\title{
BRÚQUIDOS (COLEOPTERA: BRUCHIDAE) DEL ESTADO DE MORELOS, MÉXICO
}

\author{
GUILLERMO ROMERO GÓMEZ, ${ }^{1 *}$ JESÚS ROMERO NÁPOLES, ${ }^{1}$ ARMANDO \\ BURGOS SOLORIO, ${ }^{2}$ JOSÉ LUIS CARRILLO SÁNCHEZ, ${ }^{1}$ HIRAM BRAVO \\ MOJICA $^{1}$ \& SAMUEL RAMÍREZ ALARCÓN ${ }^{3}$ \\ ${ }^{1}$ Instituto de Fitosanidad, Colegio de Postgraduados, Montecillo, 56230 Texcoco, Estado de México, \\ México. \\ ${ }^{2}$ Parasitología Vegetal del Centro de Investigaciones Biológicas, Universidad Autónoma del Estado de \\ Morelos, México. \\ ${ }^{3}$ Parasitología Agrícola, Universidad Autónoma Chapingo, Texcoco Estado de México, México. \\ *Autor de correspondencia: <gromerog@colpos.mx>
}

Romero Gómez, G., Romero Nápoles, J., Burgos Solorio, A., Carrillo Sánchez, J. L., Bravo Mojica, H. \& Ramírez Alarcón, S. 2014. Brúquidos (Coleoptera: Bruchidae) del estado de Morelos, México. Acta Zoológica Mexicana (n.s.), 30(1): 1-17.

RESUMEN. Se presenta un listado faunístico de las especies de Bruchidae y de sus plantas hospederas para el estado de Morelos. Se registraron 125 especies de brúquidos, distribuidos en 19 géneros, en donde el género Acanthoscelides presentó el mayor número de especies con 48, seguido de los géneros Merobruchus, Sennius y Amblycerus con 10 especies cada uno, y Zabrotes con 9 especies. En cuanto a las plantas hospederas se identificaron 73 especies, la familia Leguminosae presentó el mayor número con 58 plantas hospederas; la familia Convolvulaceae ocupó el segundo lugar con 8 especies. Las siguientes especies de plantas constituyeron nuevos registros de hospederos: Acacia mammifera Schltdl, Albizia adinocephala (Donn. SM.) BR. \& Rose, Albizia guachapele (Kunth) Dugan, Bauhinia variegata L., Heliocarpus pallidus Rose, Galactia striata (Jacq.) Urb. Mimosa benthamii J.F. Macbr, Mimosa leptocarpa Rose, Mimosa orthocarpa Spruce ex Benth., Senna holwayana (Rose) H.S. Irwin \& Barneby. En relación a la biodiversidad de este grupo, con la información generada en este trabajo, Morelos actualmente representa el segundo lugar en biodiversidad nacional de este grupo, con un 37.12\%; el primer lugar lo ocupa Oaxaca con $47.30 \%$.

Palabras clave: Gorgojos, plantas hospederas, diversidad.

Romero Gómez, G., Romero Nápoles, J., Burgos Solorio, A., Carrillo Sánchez, J. L., Bravo Mojica, H. \& Ramírez Alarcón, S. 2014. Bruchids (Coleoptera: Bruchidae) of the state of Morelos, Mexico. Acta Zoológica Mexicana (n.s.), 30(1): 1-17.

ABSTRACT. A list of wildlife species of Bruchidae and their host plants for the state of Morelos was performed. We recorded 125 bruchid species in 19 genera, the genus Acanthoscelides had the highest number of species with 48, followed by the genera Merobruchus, Sennius, and Amblycerus with 10 spe-

Recibido: 02/08/2012; aceptado: 24/09/2013. 
cies each, and Zabrotes with 9 species. The family Leguminosae had the highest number with 58 host plants, the family Convolvulaceae ranked second with 8 species. The following plant species: Acacia mammifera Schltdl, Albizia adinocephala (Donn. SM.) BR. \& Rose, Albizia guachapele (Kunth) Dugan, Bauhinia variegata L., Heliocarpus pallidus Rose, Galactia striata (Jacq.) Urb. Mimosa benthamii J.F. Macbr., Mimosa leptocarpa Rose, Mimosa orthocarpa Spruce ex Benth., Senna holwayana (Rose) H.S. Irwin \& Barneby, are new records as host plants for the Bruchidae family. In relation to the biodiversity in this group in Mexico, with the information generated in this work, Morelos is currently in second place with $37.12 \%$, Oaxaca is the first place with $47.30 \%$.

Key words: Seed beetles, host plants, diversity.

\section{INTRODUCCIÓN}

Romero \& Johnson (2000) estimaron que de la familia Bruchidae se reconocen en el mundo con aproximadamente 1600 especies en 62 géneros, de éstos 42 están presentes en América; sin embargo, recientemente Romero (2013) incrementó el número de especies de Bruchidae en el mundo a 1,627. Por otro lado Romero \& Johnson (2004) afirman que para México existen 334 especies distribuidas en 23 géneros. Es importante considerar que de los géneros presentes en América se incluyen cuatro que corresponden a especies introducidas del Viejo Mundo; de los géneros restantes 12 son monotípicos. Si bien el número de géneros americanos se ha incrementado por las introducciones accidentales de especies, también se tienen registros de especies de seis géneros americanos que han migrado al Viejo Mundo, ya sea de manera accidental o provocado por el comercio intenso que se realiza (Romero 2002a, Luna et al. 2006).

El avance en el conocimiento de la biodiversidad de este grupo en el mundo se ha incrementado notablemente en las últimas cuatro décadas; sin embargo, las áreas que requieren de una mayor atención son las que se encuentran en las regiones tropicales del mundo (Romero 2011). México se caracteriza por contar con dos regiones biogeográficas bien definidas, al norte con la neártica y en la parte centro-sur con la neotropical, lo que hace que sea un país con una extraordinaria biodiversidad.

El estado de Morelos se ubica en la vertiente del sur del eje neovolcánico transversal; forma parte de la cuenca del río Balsas, región situada entre aquella, la Sierra Madre del Sur y las montañas de la Mixteca, en Oaxaca. Se encuentra entre los paralelos $18^{\circ} 22^{\prime} 06^{\prime \prime}$ y $19^{\circ} 07^{\prime} 10^{\prime \prime}$ de latitud norte y los meridianos $98^{\circ} 03^{\prime} 08^{\prime \prime}$ y $98^{\circ} 30^{\prime} 08^{\prime \prime}$ de longitud oeste de Greenwich. Morelos está limitado políticamente al norte con el Estado de México y el Distrito Federal, al este y sureste con Puebla; al sur con Puebla y Guerrero; al oeste con Guerrero y Estado de México. Tiene una superficie de $4941 \mathrm{~km}^{2}$ que representa el $0.25 \%$ de la total del país (Vidal 1980). Los climas del estado, según la clasificación de Köppen modificada por García (1973), se encuentran los siguientes; a altitud menor de $1400 \mathrm{~m}$, abarcando más de la mitad del estado, se presenta el clima cálido subhúmedo con lluvias en verano. En el extremo suroeste hay dos pequeñas zonas de las estribaciones de la Sierra de Huitzuco, cerca 
de los límites con el estado de Guerrero, que presenta el clima cálido subhúmedo, pero con índice de humedad superior al clima anterior. Hacia el norte se presentan dos franjas de clima de transición entre los cálidos y los templados, la primer franja corresponde al clima semicálido con lluvias en verano. Una parte más elevada de las laderas de la Sierra, de 2200 a 2800 m, se encuentra una amplia zona de clima templado. A altitudes superiores a $2800 \mathrm{~m}$ se presenta un clima subhúmedo, corresponde a la parte más elevada de la Sierra del Ajusco, en el límite entre el D.F. y el estado de Morelos. A altitudes comprendidas entre 2800 y $4000 \mathrm{~m}$ con clima semifrío y en alturas superiores a $4000 \mathrm{~m}$, se presentan los climas fríos (Vidal 1980). De acuerdo con el criterio de Rzedowski (1978), la vegetación está compuesta principalmente por las comunidades de Bosque de Coníferas, Bosque de Quercus, Bosque Mesófilo de Montaña, Bosque Tropical Caducifolio, Pastizal, Zacatonal, Bosque de Galería y Vegetación Acuática.

En los últimos años se han desarrollado varias investigaciones con la finalidad de conocer la diversidad de este grupo de insectos en el país; por su parte Luna et al. (2002) realizaron un estudio faunístico del estado de Querétaro, encontraron un total de 57 especies y 14 géneros; en los géneros Acanthoscelides, Stator y Mimosestes encontraron el mayor número de especies; los autores indican que la diversidad de la fauna de Bruchidae representa cerca del $17.06 \%$ de las especies y el $60.86 \%$ de los géneros conocidos para México; posteriormente, Romero \& Johnson (2003), describen un nuevo género y una nueva especie de un material colectado en el cerro el Cimatario, Querétaro (Meganeltumios juani Romero and Johnson).

De Loera et al. (2006) citan para el estado de Jalisco 106 especies de brúquidos distribuidas en 14 géneros; el género Acanthoscelides resultó con el mayor número de especies (36), esto es, 34\% del total; en segundo lugar, Amblycerus, Merobruchus y Sennius con 10 especies cada uno y que equivale al $9.5 \%$ del total de especies, cada uno; el tercer lugar lo ocupó Zabrotes, Mimosestes y Megacerus, con seis especies cada género, representaron el $5.7 \%$ del total de especies, cada uno. Esta fauna del estado de Jalisco representó el $31,7 \%$ de las especies y $60.9 \%$ de los géneros registrados para México. Con respecto a sus plantas hospederas, del total de brúquidos el $46.6 \%$ registró una planta asociada, $14.56 \%$ tuvo dos especies de plantas asociadas, 9.7\% tres, y el 5.84\% cuatro o más hospederas; en tanto que para el resto (23.3\%) no encontraron información sobre sus hospederos.

Luna et al. (2006) realizaron un estudio parcial sobre la diversidad de brúquidos en el estado de Guerrero; estudiaron la bruquidofauna de la localidad de Platanillo, municipio de Iguala. En este estudio se reportan 5 géneros con 16 especies de brúquidos asociados a 13 plantas hospederas.

De acuerdo con Romero (2011), en una investigación sobre brúquidos del estado de Veracruz se registraron 123 especies, que representó el 36.82\% de diversidad de brúquidos en la República Mexicana; estas especies se encuentran agrupadas en 16 
géneros (Cuadro 1). Con respecto a las plantas hospederas se registraron 42 especies, entre las que abundaron las leguminosas.

De la Cruz et al. (2013) en su investigación realizada para conocer la diversidad de la familia Bruchidae en el estado de Tabasco identificó 30 especies de plantas como hospederas de brúquidos; en cuanto a los insectos, se determinaron 14 géneros y 50 especies de brúquidos.

Burgos \& Trejo (2001) realizaron un listado preliminar de los coleópteros registrados para el estado de Morelos, en el cual se registraron 38 especies de brúquidos. El género Acanthoscelides presentó el mayor número con ocho especies, seguido del género Zabrotes con siete especies.

El trabajo más reciente sobre la diversidad de brúquidos en Morelos fue realizado por Romero \& Westcott (2011) en la Reserva de la Biosfera Sierra de Huautla; los autores registraron para la reserva un total de 72 especies, incluidas en 13 géneros, cuatro tribus y dos subfamilias. La sola reserva acumuló el $21.56 \%$ de la biodiversidad mexicana. Con respecto a las plantas hospederas, los autores registraron un total de 36 especies, mismas que se encontraron asociadas a 36 especies de brúquidos; las plantas incluyeron principalmente a Leguminosae $(86.15 \%)$, Boraginaceae $(2.77 \%)$, Sterculiaceae $(2.77 \%)$, Tiliaceae $(2.77 \%)$, Convolvulaceae $(2.77 \%)$ y Lamiaceae (2.77\%). En el Cuadro 1 se pueden observar las comparaciones de porcentajes de géneros y especies de Bruchidae de los trabajos realizados sobre riqueza faunística de este grupo en México.

Aunque se han realizado listados faunísticos en seis estados de la República Mexicana, todavía falta bastante para poder tener una evaluación completa de la biodiversidad de esta familia de insectos en el país. Con respecto al estado de Morelos, si bien se cuenta con un estudio en una reserva del estado, es importante tener información sobre el resto del estado, por tal motivo la investigación tuvo por objetivos: a) Elaborar un listado faunístico de brúquidos del estado de Morelos, y b) Conocer el espectro alimentario de estos insectos.

\section{MATERIAL Y MÉTODOS}

Se realizaron recolecciones intensivas de brúquidos en los años 2010 y 2011 tratando de cubrir los diferentes tipos de vegetación que se presentan en el estado de Morelos. Se utilizaron dos métodos de colecta: 1) colecta directa, que consistió en utilizar una red entomológica de golpeo, dando de 10-20 redadas sobre la vegetación, posteriormente el contenido de la red se vació en una bolsa de plástico para facilitar la separación de los brúquidos, mismos que se depositaron en tubos de ensayo con alcohol al $70 \%$ y se anexaron etiquetas con los datos de colecta; 2) colecta de semillas, para este tipo de recolección se cortaban directamente frutos y/o vainas, mismas que se colocaban en bolsas de papel anexando etiquetas con los datos respectivos de colecta; el material se transportó al laboratorio de Taxonomía del Colegio de Postgraduados 


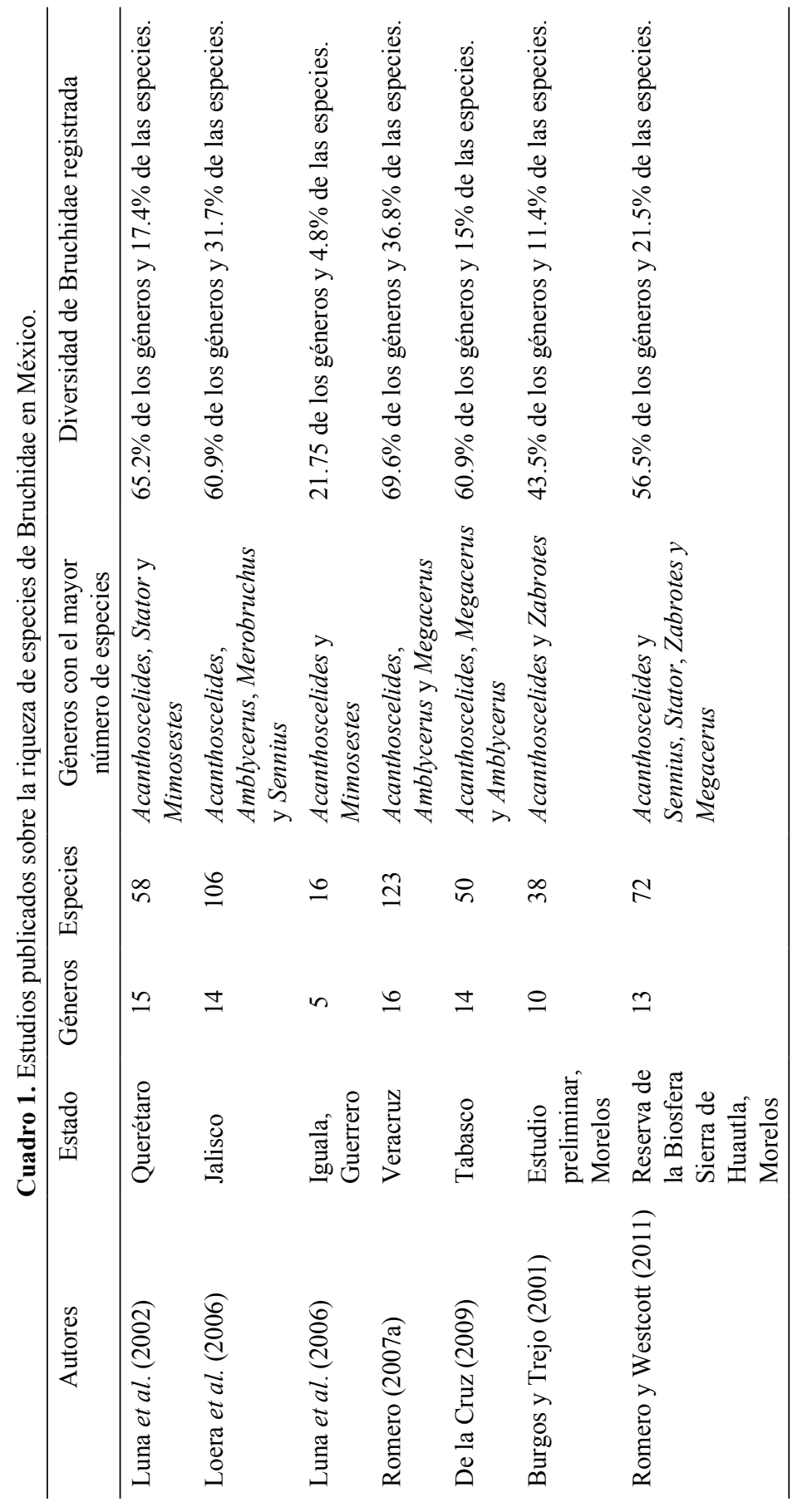


para esperar los insectos emergidos. Posteriormente los adultos al emerger de los frutos y/o vainas se preservaron en alcohol al $70 \%$.

Para la determinación de las especies de insectos se requirió de la extracción de la genitalia de los machos; para lo cual se siguió la técnica propuesta por Kingsolver (1970) y para la interpretación de las estructuras de la genitalia se utilizó la nomenclatura propuesta por Romero \& Johnson (1999). Las claves empleadas para la determinación de las especies se indican a continuación: Acanthoscelides (Johnson 1990), Amblycerus (Romero et al. 1996), Callosobruchus (Kingsolver 2004), Caryedes (Kingsolver \& Whitehead 1974b), Caryedon (Johnson et al. 2004), Ctenocolum (Kingsolver \& Whitehead 1974a), Gibbobruchus (Whitehead \& Kingsolver 1975), Megacerus(Teran \& Kingsolver 1977), Meibomeus (Kingsolver \& Whitehead 1976, Romero 2002b), Merobruchus (Kingsolver 1988), Mimosestes (Kingsolver \& Johnson 1978), Sennius (Johnson \& Kingsolver 1973), Stator (Johnson \& Kingsolver 1976), Zabrotes (Romero \& Johnson 2000). La determinación de las plantas se hizo por comparación en el herbario de la Especialidad de Botánica del Colegio de Postgraduados (CHAPA). Todo el material de Bruchidae se encuentra depositado en la colección Entomológica del Instituto de Fitosanidad (CEAM).

El listado faunístico final para el estado de Morelos se obtuvo al fusionar la información generada en este trabajo y la información presente en la base de datos BRUCOL (Romero \& Johnson, 2002a) sobre el estado de Morelos, es importante comentar que la información que actualmente tiene la base de datos proviene de literatura especializada y de la consulta de las colecciones más completas de este grupo de insectos.

\section{RESULTADOS}

Para el estado de Morelos se registraron 125 especies de brúquidos (Cuadro 1). Los cuales están distribuidos en los siguientes 19 géneros: Acanthoscelides (48 especies), Amblycerus (10), Merobruchus (10), Sennius (10), Zabrotes (9), Megacerus (8), Stator (7), Mimosestes (6), Meibomeus (5), Algarobius (2), Dalibruchus (2). Callosobruchus (1), Caryedes (1), Caryedon (1), Caryobruchus (1), Cosmobruchus (1), Ctenocolum (1), Margaritabruchus (1) y Specularius (1). Al comparar la riqueza entre la Reserva de la Biosfera Sierra de Huautla (RBSH) con respecto al total de las especies registradas en el estado de Morelos, se encontró que el 58.06\% está representado en la RBSH. Morelos como estado concentra un 37.4\% de las especies del país, mientras que para el mundo un $7.7 \%$. Actualmente, los tres estados mexicanos que tienen la mayor diversidad de especies de brúquidos son: Oaxaca (47.3\%), Morelos (37.4\%) y Veracruz (36.8\%).

Como plantas hospederas se registraron ocho familias con un total de 73 especies. La familia Leguminosae presentó el mayor número de especies con 58 , seguida de Convolvulaceae con ocho, Sterculiaceae con dos y las familias Arecaceae, Astera- 
ceae, Boraginaceae, Malvaceae y Tiliaceae con una especie. Al comparar el número de hospederos entre la RBSH y el estado de Morelos en su totalidad, se encontró que existió un incremento de más del $270.4 \%$; en tanto que con el número de especies de brúquidos se registró un incremento de más del 174\% (Cuadro 2).

Las especies de Bruchidae más polífagas resultaron ser Megacerus tricolor (Suffrian) al alimentarse de seis diferentes especies de plantas, aunque todas en la familia Convolvulaceae; para Mimosestes mimosae F. y Stator limbatus (Horn) se registraron cinco plantas, Acanthoscelides biustulus (Fall) y Merobruchus insolitus (Sharp) cuatro plantas, Acanthoscelides mexicanus (Sharp), A. obrienorum Johnson, Megacerus maculiventris (Fåhraeus), Sennius lebasi (Fåhraeus), Stator sordidus (Horn), y $S$. vittatithorax (Pic) tres plantas. Por el contrario las especies oligófagas que se registraron fueron: Acanthoscelides guazumae Johnson \& Kingsolver, A. kingsolveri Johnson, A. megacornis Kingsolver, A. mimosicola Johnson, A. obtetus (Say), Megacerus impiger (Horn), Sennius rufomaculatus (Motschulsky) y Zabrotes subfasciatus (Boheman); en tanto que las especies monófagas en total sumaron 51 especies (Cuadro 2). De 55 especies no se tiene determinadas sus plantas hospederas para el estado de Morelos, aunque para algunos de ellos existen registros de plantas hospederas para otros estados o países.

Las plantas hospederas con mayor número de especies de Bruchidae asociados fueron las siguientes; Phaseolus vulgaris L. y Lysiloma acapulcense (Kunth) Benth., cuatro brúquidos; Acacia farnesiana (L.) Willd., Albizia adinocephala e Ipomoea triloba L., tres especies; Acacia pennatula (Schldl. \& Cham.) Benth., Albizia guachapele, Bauhinia variegata, Chamaecrista nictitans (L.) Moench, Conzattia multiflora (B.L. Rob.) Standl., Guazuma tomentosa Kunth, Guazuma ulmifolia Lam., Ipomoea cholulensis Kunth, Ipomoea tricolor Cav., Lysiloma divaricatum (Jacq.) J. F. Macbr., Merremia quinquefolia (L.) Hallierf., Mimosa galeottii Benth., Phaseolus lunatus L., Pithecellobium dulce (Roxb.) Benth., Senna hirsuta (L.) H. S. Irwin \& Barneby, S. holwayana y Senna occidentalis (L.) Link, dos brúquidos y las demás plantas (51 especies) sólo presentaron una especie de brúquido.

Del total de las especies de brúquidos reportadas, de 54 de ellos no se registró información de su especie de planta hospedera para el estado de Morelos; sin embargo, si existen reportes de especies hospederas para ellos en otros estados de la Republica Mexicana o en otros países; de las siguientes siete especies aún se desconocen sus hospederos: Acanthoscelides burkei Johnson, A. leisneri Johnson, A. luteus Johnson, A. oaxaca Johnson, A. yecora Johnson, Amblycerus cuernavacensis Romero, Johnson \& Kingsolver y Zabrotes densus (Horn).

\section{DISCUSIÓN}

El estado de Morelos pese a su tamaño, su topografía le permite presentar una amplia variedad de comunidades vegetales, mismas que se encuentran muy íntimamente re- 
Romero et al.: Brúquidos de Morelos, México

Cuadro 2. Especies de brúquidos y sus plantas hospederas en el estado de Morelos.

\begin{tabular}{|c|c|c|}
\hline Especies de brúquidos & Plantas hospederas & Familia \\
\hline Acanthoscelides amabilis Johnson, 1983. & $\begin{array}{l}\text { Rhynchosia discolor M. Martens \& } \\
\text { Galeotti }\end{array}$ & Leguminosae \\
\hline Acanthoscelides anoditus Johnson, 1983 & $\mathrm{CD}$ & $\mathrm{CD}$ \\
\hline $\begin{array}{l}\text { Acanthoscelides argillaceus (Sharp), } \\
1885\end{array}$ & Phaseolus lunatus $\mathrm{L}$. & Leguminosae \\
\hline \multicolumn{3}{|l|}{ Acanthoscelides } \\
\hline biustulus (Fall), 1910 & Desmodium densiflorum Hemsl. & \\
\hline \multicolumn{3}{|l|}{ Desmodium hartwegianum Hemsl. } \\
\hline \multicolumn{3}{|l|}{$\begin{array}{l}\text { Desmodium hartwegianum var. } \\
\text { hartwegianum Hemsl. }\end{array}$} \\
\hline Desmodium sericophyllum Schltdl. & Leguminosae & \\
\hline Acanthoscelides burkei Johnson, 1983 & ND & ND \\
\hline $\begin{array}{l}\text { Acanthoscelides camerinoi Romero, } \\
2011\end{array}$ & $\begin{array}{l}\text { Senna holwayana (Rose) H.S. Irwin \& } \\
\text { Barneby }\end{array}$ & Leguminosae \\
\hline Acanthoscelides chiricahuae (Fall), 1910 & Mimosa orthocarpa Spruce ex Benth. & Leguminosae \\
\hline $\begin{array}{l}\text { Acanthoscelides clandestinus } \\
\text { (Motschulsky), } 1874\end{array}$ & $\mathrm{CD}$ & $\mathrm{CD}$ \\
\hline $\begin{array}{l}\text { Acanthoscelides clitellarius (Fåhraeus), } \\
1839\end{array}$ & Mimosa leptocarpa Rose & Leguminosae \\
\hline Acanthoscelides cordifer (Sharp), 1885 & $\mathrm{CD}$ & $\mathrm{CD}$ \\
\hline Acanthoscelides cornis Johnson, 1983 & Heliocarpus pallidus Rose & Tiliaceae \\
\hline $\begin{array}{l}\text { Acanthoscelides cuernavaca Johnson, } \\
1983\end{array}$ & ND & ND \\
\hline $\begin{array}{l}\text { Acanthoscelides desmodicola Johnson, } \\
1983\end{array}$ & $\mathrm{CD}$ & $\mathrm{CD}$ \\
\hline $\begin{array}{l}\text { Acanthoscelides desmoditus Johnson, } \\
1983\end{array}$ & $\mathrm{CD}$ & $\mathrm{CD}$ \\
\hline Acanthoscelides difficilis (Sharp), 1885 & $\begin{array}{l}\text { Mimosa albida var. strigosa (Willd.) } \\
\text { B.L. Rob. }\end{array}$ & Leguminosae \\
\hline $\begin{array}{l}\text { Acanthoscelides eriosemicola Johnson, } \\
1983\end{array}$ & $\mathrm{CD}$ & $\mathrm{CD}$ \\
\hline $\begin{array}{l}\text { Acanthoscelides flavescens (Fåhraeus), } \\
1839\end{array}$ & $\mathrm{CD}$ & $\mathrm{CD}$ \\
\hline Acanthoscelides griseolus (Fall), 1910 & Sesbania sp. & Leguminosae \\
\hline $\begin{array}{l}\text { Acanthoscelides guazumae Johnson \& } \\
\text { Kingsolver, } 1971\end{array}$ & $\begin{array}{l}\text { Guazuma tomentosa Kunth, Guazuma } \\
\text { ulmifolia Lam. }\end{array}$ & Sterculiaceae \\
\hline $\begin{array}{l}\text { Acanthoscelides howdenorum Johnson, } \\
1983\end{array}$ & $\mathrm{CD}$ & $\mathrm{CD}$ \\
\hline
\end{tabular}


Cuadro 2. Continúa.

\begin{tabular}{|c|c|c|}
\hline Especies de brúquidos & Plantas hospederas & Familia \\
\hline $\begin{array}{l}\text { Acanthoscelides johnsoni Kingsolver, } \\
1980\end{array}$ & $\mathrm{CD}$ & $\mathrm{CD}$ \\
\hline $\begin{array}{l}\text { Acanthoscelides kingsolveri Johnson, } \\
1974\end{array}$ & Indigofera sp. & \\
\hline Indigofera suffruticosa Mill. & Leguminosae & \\
\hline Acanthoscelides leisneri Johnson, 1983 & ND & ND \\
\hline Acanthoscelides luteus Johnson, 1983 & ND & ND \\
\hline $\begin{array}{l}\text { Acanthoscelides macrophthalmus } \\
\text { (Schaeffer, 1907) }\end{array}$ & $\begin{array}{l}\text { Leucaena esculenta (Moc. \& Sesse ex } \\
\text { DC.) Benth. }\end{array}$ & Leguminosae \\
\hline Acanthoscelides mankinsi Johnson, 1983 & Leucaena sp. & Leguminosae \\
\hline Acanthoscelides mazatlan Johnson, 1983 & $\mathrm{CD}$ & $\mathrm{CD}$ \\
\hline $\begin{array}{l}\text { Acanthoscelides megacornis Kingsolver, } \\
1980\end{array}$ & $\begin{array}{l}\text { Aeschynomene americana } \mathrm{L} . \\
\text { Desmodium } \mathrm{sp} .\end{array}$ & Leguminosae \\
\hline $\begin{array}{l}\text { Acanthoscelides mexicanus (Sharp), } \\
1885\end{array}$ & $\begin{array}{l}\text { Mimosa benthamii J.F. Macbr. } \\
\text { Mimosa galeottii Benth., Mimosa } \\
\text { polyantha Benth. }\end{array}$ & Leguminosae \\
\hline $\begin{array}{l}\text { Acanthoscelides mimosicola Johnson, } \\
1983\end{array}$ & Lysiloma acapulcense (Kunth) Benth., & \\
\hline Mimosa sp. & Leguminosae & \\
\hline Acanthoscelides modestus (Sharp), 1885 & $\mathrm{CD}$ & $\mathrm{CD}$ \\
\hline Acanthoscelides mundulus (Sharp), 1885 & Nissolia fruticosa Jacq. & Leguminosae \\
\hline Acanthoscelides oaxaca Johnson, 1983 & $\mathrm{CD}$ & $\mathrm{CD}$ \\
\hline $\begin{array}{l}\text { Acanthoscelides obrienorum Johnson, } \\
1970\end{array}$ & $\begin{array}{l}\text { Acacia mammifera Schltdl., Cassia } \\
\text { sp., }\end{array}$ & \\
\hline Senna sp. & Leguminosae & \\
\hline Acanthoscelides obtectus (Say), 1831 & $\begin{array}{l}\text { Phaseolus coccineus L., Phaseolus } \\
\text { vulgaris L. }\end{array}$ & Leguminosae \\
\hline $\begin{array}{l}\text { Acanthoscelides obvelatus Bridwell, } \\
1942\end{array}$ & Phaseolus vulgaris L. & Leguminosae \\
\hline $\begin{array}{l}\text { Acanthoscelides pedicularius (Sharp), } \\
1885\end{array}$ & $\mathrm{CD}$ & $\mathrm{CD}$ \\
\hline Acanthoscelides pertinax (Sharp), 1885 & Galactia striata (Jacq.) Urb. & Leguminosae \\
\hline Acanthoscelides puellus (Sharp), 1885 & $\mathrm{CD}$ & $\mathrm{CD}$ \\
\hline $\begin{array}{l}\text { Acanthoscelides pusillimus (Sharp), } \\
1885\end{array}$ & $\mathrm{CD}$ & $\mathrm{CD}$ \\
\hline $\begin{array}{l}\text { Acanthoscelides quadridentatus } \\
\text { (Schaeffer), } 1907\end{array}$ & Mimosa galeottii Benth. & Leguminosae \\
\hline
\end{tabular}


Cuadro 2. Continúa.

\begin{tabular}{|c|c|c|}
\hline Especies de brúquidos & Plantas hospederas & Familia \\
\hline $\begin{array}{l}\text { Acanthoscelides rhynchosiestes Johnson, } \\
1983\end{array}$ & Rhynchosia pyramidalis (Lam.) Urb. & Leguminosae \\
\hline Acanthoscelides sanfordi Johnson, 1983 & Pachyrhizus erosus (L.) Urb. & Leguminosae \\
\hline $\begin{array}{l}\text { Acanthoscelides speciosus (Schaeffer), } \\
1907\end{array}$ & $\mathrm{CD}$ & $\mathrm{CD}$ \\
\hline Acanthoscelides stylifer (Sharp), 1885 & $\mathrm{CD}$ & $\mathrm{CD}$ \\
\hline $\begin{array}{l}\text { Acanthoscelides subaequalis Johnson, } \\
1970\end{array}$ & Abutilon trisulcatum (Jacq.) Urban & Malvaceae \\
\hline $\begin{array}{l}\text { Acanthoscelides triumfettae Kingsolver, } \\
1980\end{array}$ & $\mathrm{CD}$ & $\mathrm{CD}$ \\
\hline Acanthoscelides yecora Johnson, 1983 & ND & ND \\
\hline Algarobius johnsoni Kingsolver, 1986 & $\begin{array}{l}\text { Havardia acatlensis (Benth.) Britton } \\
\& \text { Rose }\end{array}$ & Leguminosae \\
\hline Algarobius nicoya Kingsolver, 1986 & Prosopis juliflora (SW.) DC. & Leguminosae \\
\hline Amblycerus alternates (Pic), 1954 & ND & ND \\
\hline Amblycerus barcenae (Dugés), 1880 & ND & ND \\
\hline Amblycerus cistelinus (Gyllenhal), 1833 & Guazuma ulmifolia Lam. & Sterculiaceae \\
\hline $\begin{array}{l}\text { Amblycerus cuernavacensis Romero, } \\
\text { Johnson \& Kingsolver, } 1996\end{array}$ & ND & ND \\
\hline $\begin{array}{l}\text { Amblycerus guazumicola Kingsolver \& } \\
\text { Johnson, } 1971\end{array}$ & Guazuma tomentosa Kunth & Sterculiaceae \\
\hline Amblycerus montalvoi Romero, 2011 & Cordia morelosana Standl. & Boraginaceae \\
\hline Amblycerus obscurus (Sharp), 1885 & $\mathrm{CD}$ & $\mathrm{CD}$ \\
\hline Amblycerus perfectus (Sharp), 1885 & $\mathrm{CD}$ & $\mathrm{CD}$ \\
\hline Amblycerus scutellaris (Sharp), 1885 & $\mathrm{CD}$ & $\mathrm{CD}$ \\
\hline Amblycerus spondiae Kingsolver, 1980 & $\mathrm{CD}$ & $\mathrm{CD}$ \\
\hline $\begin{array}{l}\text { Callosobruchus maculatus (Fabricius), } \\
1775\end{array}$ & Cicer arietinum $\mathrm{L}$. & Leguminosae \\
\hline Caryedes longicollis (Fåhraeus), 1839 & $\mathrm{CD}$ & $\mathrm{CD}$ \\
\hline Caryedon serratus (Olivier), 1790 & $\mathrm{CD}$ & $\mathrm{CD}$ \\
\hline Caryobruchus rubidus (Chevrolat), 1877 & Brahea sp. & Arecaceae \\
\hline Cosmobruchus russelli Bridwell, 1931 & $\mathrm{CD}$ & $\mathrm{CD}$ \\
\hline $\begin{array}{l}\text { Ctenocolum janzeni Kingsolver \& } \\
\text { Whitehead, } 1974\end{array}$ & Lonchocarpus rugosus Benth. & Leguminosae \\
\hline Dahlibruchus conradti Bridwell, 1931 & Dahlia sp. & Asteraceae \\
\hline $\begin{array}{l}\text { Dahlibruchus nezahualcoyotli Romero } \\
\text { \& Romero, } 2011\end{array}$ & Dahlia sp. & Asteraceae \\
\hline
\end{tabular}


Cuadro 2. Continúa.

\begin{tabular}{|c|c|c|}
\hline Especies de brúquidos & Plantas hospederas & Familia \\
\hline $\begin{array}{l}\text { Margaritabruchus cherylae Romero \& } \\
\text { Johnson, } 2001 .\end{array}$ & $\begin{array}{l}\text { Indigofera densiflora M. Martens \& } \\
\text { Galeotti }\end{array}$ & Leguminosae \\
\hline $\begin{array}{l}\text { Megacerus alabani Teran \& Kingsolver, } \\
1977\end{array}$ & $\mathrm{CD}$ & $\mathrm{CD}$ \\
\hline Megacerus callirhipis (Sharp), 1885 & Ipomoea parasitica (Kunth) G. Don & Convolvulaceae \\
\hline Megacerus contaminates (Sharp), 1885 & $\mathrm{CD}$ & $\mathrm{CD}$ \\
\hline Megacerus cubiculus (Casey), 1884 & Ipomoea sp. & Convolvulaceae \\
\hline Megacerus impiger (Horn), 1873 & $\begin{array}{l}\text { Ipomoea triloba L., Merremia } \\
\text { quinquefolia (L.) Hallier f. }\end{array}$ & Convolvulaceae \\
\hline Megacerus lherminieri (Fåhraeus), 1839 & $\mathrm{CD}$ & $\mathrm{CD}$ \\
\hline $\begin{array}{l}\text { Megacerus maculiventris (Fåhraeus), } \\
1839\end{array}$ & $\begin{array}{l}\text { Ipomoea cholulensis Kunth, Ipomoea } \\
\text { tricolor Cav., Ipomoea triloba L. }\end{array}$ & Convolvulaceae \\
\hline Megacerus tricolor (Suffrian), 1870 & $\begin{array}{l}\text { Ipomoea cholulensis Kunth, Ipomoea } \\
\text { hederifolia L., Ipomoea purpurea (L.) } \\
\text { Roth }\end{array}$ & \\
\hline \multicolumn{3}{|l|}{$\begin{array}{l}\text { Ipomoea tricolor Cav., Ipomoea triloba } \\
\text { L. }\end{array}$} \\
\hline $\begin{array}{l}\text { Merremia quinquefolia (L.) Hallier f. } \\
\text { Meibomeus desmoportheus }\end{array}$ & Convolvulaceae & \\
\hline Kingsolver\&Whitehed, 1976 & $\mathrm{CD}$ & $\mathrm{CD}$ \\
\hline $\begin{array}{l}\text { Meibomeus hidalgoi } \\
\text { Kingsolver\&Whitehed, } 1976\end{array}$ & Desmodium sp. & Leguminosae \\
\hline Meibomeus ptinoides (Sharp), 1885 & $\mathrm{CD}$ & $\mathrm{CD}$ \\
\hline Meibomeus serraticulus (Sharp), 1885 & Desmodium sp. & Leguminosae \\
\hline Meibomeus surrubresus (Pic), 1939 & $\mathrm{CD}$ & $\mathrm{CD}$ \\
\hline Merobruchus hastatus Kingsolver, 1988 & $\begin{array}{l}\text { Albizia adinocephala (Donn. SM.) BR. } \\
\& \text { Rose }\end{array}$ & Leguminosae \\
\hline Merobruchus insolitus (Sharp), 1885 & $\begin{array}{l}\text { Acacia farnesiana (L.) Willd, Albizia } \\
\text { occidentalis Brand., Lysiloma } \\
\text { acapulcense (Kunth) Benth., Lysiloma } \\
\text { divaricatum (Jacq.) J.F. Macbr. }\end{array}$ & Leguminosae \\
\hline Merobruchus julianus (Horn), 1894 & Acacia sp. & Leguminosae \\
\hline Merobruchus knulli (White), 1941 & $\mathrm{CD}$ & $\mathrm{CD}$ \\
\hline Merobruchus placidus (Horn), 1873 & $\mathrm{CD}$ & $\mathrm{CD}$ \\
\hline $\begin{array}{l}\text { Merobruchus santarosae Kingsolver, } \\
1980\end{array}$ & Acacia coulteri A. Gray & Leguminosae \\
\hline Merobruchus terani Kingsolver, 1980 & Albizia guachapele (Kunth) Dugand & Leguminosae \\
\hline
\end{tabular}


Cuadro 2. Continúa.

\begin{tabular}{|c|c|c|}
\hline Especies de brúquidos & Plantas hospederas & Familia \\
\hline $\begin{array}{l}\text { Merobruchus triacanthus Kingsolver, } \\
1988\end{array}$ & Acacia sp. & Leguminosae \\
\hline Merobruchus vacillator (Sharp), 1885 & $\begin{array}{l}\text { Lysiloma divaricatum (Jacq.) J.F. } \\
\text { Macbr. }\end{array}$ & Leguminosae \\
\hline $\begin{array}{l}\text { Merobruchus xanthopygus Kingsolver, } \\
1988\end{array}$ & $\mathrm{CD}$ & $\mathrm{CD}$ \\
\hline $\begin{array}{l}\text { Mimosestes acaciestes Kingsolver \& } \\
\text { Johnson, } 1978\end{array}$ & Acacia sp. & Leguminosae \\
\hline Mimosestes humeralis Gyllenhal), 1833 & $\begin{array}{l}\text { Acacia pennatula (Schldl. \& Cham.) } \\
\text { Benth. }\end{array}$ & Leguminosae \\
\hline $\begin{array}{l}\text { Mimosestes janzeni Kingsolver \& } \\
\text { Johnson, } 1978\end{array}$ & $\mathrm{CD}$ & $\mathrm{CD}$ \\
\hline Mimosestes mimosa (Fabricius), 1781 & $\begin{array}{l}\text { Acacia cochliacantha Humb. \& } \\
\text { Bonpl., Acacia farnesiana (L.) Willd., } \\
\text { Acacia pennatula (Schldl. \& Cham.) } \\
\text { Benth. }\end{array}$ & \\
\hline $\begin{array}{l}\text { Bauhinia variegata L., Conzattia } \\
\text { multiflora (B.L. Rob.) Standl. }\end{array}$ & Leguminosae & \\
\hline $\begin{array}{l}\text { Mimosestes nubigens (Motschulsky), } \\
1874\end{array}$ & Acacia farnesiana (L.) Willd. & Leguminosae \\
\hline Mimosestes protractus (Horn), 1873 & $\mathrm{CD}$ & $\mathrm{CD}$ \\
\hline $\begin{array}{l}\text { Sennius atripectus Johnson \& } \\
\text { Kingsolver } 1973\end{array}$ & Cassia sp. & Leguminosae \\
\hline $\begin{array}{l}\text { Sennius auricomus Johnson \& } \\
\text { Kingsolver } 1973\end{array}$ & $\begin{array}{l}\text { Senna holwayana (Rose) H.S. Irwin \& } \\
\text { Barneby }\end{array}$ & Leguminosae \\
\hline $\begin{array}{l}\text { Sennius ensiculus Johnson \& Kingsolver } \\
1973\end{array}$ & Chamaecrista nictitans (L.) Moench & Leguminosae \\
\hline Sennius lebasi (Fåhraeus), 1839 & $\begin{array}{l}\text { Senna hirsuta (L.) H.S. Irwin \& } \\
\text { Barneby, }\end{array}$ & \\
\hline $\begin{array}{l}\text { Senna occidentalis (L.) Link, Sesbania } \\
\text { herbacea (Mill.) McVaugh }\end{array}$ & Leguminosae & \\
\hline $\begin{array}{l}\text { Sennius leucostauros Johnson \& } \\
\text { Kingsolver, } 1973\end{array}$ & $\mathrm{CD}$ & $\mathrm{CD}$ \\
\hline Sennius medialis (Sharp), 1885 & $\begin{array}{l}\text { Senna obtusifolia (L.) H.S. Irwin \& } \\
\text { Barneby }\end{array}$ & Leguminosae \\
\hline Sennius militaris (Sharp), 1885 & $\mathrm{CD}$ & $\mathrm{CD}$ \\
\hline Sennius morosus (Sharp), 1885 & Lysiloma acapulcense (Kunth) Benth. & Leguminosae \\
\hline $\begin{array}{l}\text { Sennius rufomaculatus (Motschulsky), } \\
1874\end{array}$ & $\begin{array}{l}\text { Senna hirsuta (L.) H.S. Irwin \& } \\
\text { Barneby }\end{array}$ & \\
\hline
\end{tabular}


Cuadro 2. Continúa.

\begin{tabular}{|c|c|c|}
\hline Especies de brúquidos & Plantas hospederas & Familia \\
\hline Senna occidentalis (L.) Link. & Leguminosae & \\
\hline Sennius simulans (Schaeffer), 1907 & Chamaecrista nictitans (L.) Moench & Leguminosae \\
\hline Specularius impressithorax Pic, 1932 & Erythrina coralloides DC. & Leguminosae \\
\hline $\begin{array}{l}\text { Stator dissimilis Johnson \& Kingsolver, } \\
1976\end{array}$ & $\mathrm{CD}$ & $\mathrm{CD}$ \\
\hline $\begin{array}{l}\text { Stator huautlae Romero \& Johnson, } \\
2004\end{array}$ & Salvia sessei Benth. & Leguminosae \\
\hline Stator limbatus (Horn), 1873 & $\begin{array}{l}\text { Acacia sp., Albizia adinocephala } \\
\text { (Donn. SM.) BR. \& Rose, Havardia } \\
\text { acatlensis (Benth.) Britton \& Rose, } \\
\text { Lysiloma acapulcense (Kunth) Benth., } \\
\text { Pithecellobium dulce (Roxb.) Benth. }\end{array}$ & Leguminosae \\
\hline Stator pruininus (Horn), 1873 & Acacia sp. & Leguminosae \\
\hline Stator sordidus (Horn), 1873 & $\begin{array}{l}\text { Bauhinia variegata L., } \\
\text { Conzattia multiflora (B.L. Rob.) } \\
\text { Standl.,Pithecellobium dulce (Roxb.) } \\
\text { Benth. }\end{array}$ & Leguminosae \\
\hline Stator vachelliae Bottimer, 1973 & $\mathrm{CD}$ & $\mathrm{CD}$ \\
\hline Stator vittatithorax (Pic), 1930 & $\begin{array}{l}\text { Acacia picachensis Brandegee, Albizia } \\
\text { adinocephala (Donn. SM.) BR. \& } \\
\text { Rose, }\end{array}$ & \\
\hline Albizia guachapele (Kunth) Dugand & Leguminosae & \\
\hline $\begin{array}{l}\text { Zabrotes achiote Romero \& Johnson, } \\
2000\end{array}$ & $\mathrm{CD}$ & $\mathrm{CD}$ \\
\hline Zabrotes chavesi Kingsolver, 1980 & $\mathrm{CD}$ & $\mathrm{CD}$ \\
\hline Zabrotes densus (Horn), 1885 & ND & ND \\
\hline Zabrotes obliteratus (Horn), 1885 & $\mathrm{CD}$ & $\mathrm{CD}$ \\
\hline Zabrotes planifrons (Horn), 1885 & $\mathrm{CD}$ & $\mathrm{CD}$ \\
\hline Zabrotes propinquus (Horn), 1885 & $\mathrm{CD}$ & $\mathrm{CD}$ \\
\hline Zabrotes spectabilis Horn, 1885 & $\mathrm{CD}$ & $\mathrm{CD}$ \\
\hline Zabrotes subfasciatus (Boheman), 1833 & $\begin{array}{l}\text { Phaseolus lunatus L., Phaseolus } \\
\text { vulgaris L. }\end{array}$ & Leguminosae \\
\hline $\begin{array}{l}\text { Zabrotes sylvestris Romero \& Johnson, } \\
1999\end{array}$ & Phaseolus vulgaris L. & Leguminosae \\
\hline
\end{tabular}

ND: refiere a que el insecto no cuenta con información sobre plantas hospederas para el estado de Morelos.

CD: indica que existen registros de plantas hospederas para el insecto para otros estados y/o países. 
lacionadas con una amplia gama de insectos (Rzedowski 1978, Vidal 1980). Aunque, hasta el momento se cuenta con una muy buena aproximación del número de especies de brúquidos (Romero 2011), no dudamos que todavía se pueda incrementar el número de registros, nuevas especies, y aún géneros nuevos, no sólo a nivel estatal sino también del país. Otra parte de información sobre la que se requiere trabajar, es sobre el conocimiento de las plantas hospederas, todavía de muchas especies de brúquidos no se conocen éstas, aunque Romero y Johnson (2002a) registran una buena aproximación sobre el total de plantas hospederas para la familia Bruchidae, que asciende a 1302 especies. Según Romero (2002a) la mayoría de los brúquidos son monófagos; sin embargo, algunas especies son oligófagas y muy pocas polífagas. Los brúquidos que se alimentan de varias especies o géneros de plantas tienen una amplia distribución geográfica en comparación con aquellas que se alimentan de una o pocas hospederas.

En este grupo de insectos sólo algunas especies han llegado a constituirse en serias plagas, debido a que se han especializado en el ataque de productos almacenados, como es el caso de los gorgojos del frijol, Acanthoscelides obtectus y Zabrotes subfasciatus que causan daños sólo en su estado larvario y las cuales se colectaron durante este estudio en semillas de plantas silvestres. De acuerdo con Romero y Johnson (1999) existe otra especie muy relacionada a Z. subfasciatus, Z. sylvestris Romero \& Johnson que se alimenta de las formas silvestres de Phaseolus vulgaris. Otra especie que puede ser se importancia económica es Caryedon serratus (Olivier), a la cual se le registró alimentándose de semillas de tamarindo (Tamarindus indica $L$.$) ; se trata de una especie exótica que se introdujo de las partes tropicales del Viejo$ Mundo, y que ya se encuentra completamente adaptado a nuestro país y en general al Continente Americano; según Romero y Johnson (2002b) se trata de una especie polífaga ya que se le ha registrado en varias especies de los siguientes géneros: Acacia (12 especies), Bauhinia (7 especies), Cassia (13 especies), Caesalpinia, Piliostigma y Prosopis (8 especies).

Entre las especies de Bruchidae que se colectaron y que podrían tener en el futuro alguna importancia económica se encuentran Acanthoscelides guazumae y Amblycerus guazumicola Kingsolver \& Johnson que se registraron alimentándose de semillas del árbol denominado cuahulote (Guazuma spp.), del cual se sabe presenta propiedades medicinales. Para el caso de las especies de Indigofera, en la literatura se reconoce su importancia industrial debido a que se utilizan para extraer el colorante añil; al respecto, en esta investigación se detectaron las especies Acanthoscelides kingsolveri en semillas de Indigofera suffruticosa Mill. y Margaritabruchus cherylae Romero \& Johnson en semillas de I. densiflora M. Martens \& Galleotti.

El grupo de plantas que se encuentra más estrechamente relacionado con la familia Bruchidae son las leguminosas con 58 especies; al respecto Nadal et al. (2004), afirma que esta familia se encuentra entre el grupo de plantas que representa la impor- 
tancia económica y ecológica más alta de nuestro planeta; dicha importancia radica su utilidad en la alimentación humana y animal, jardinería y ornamentación, artesanías, construcción, cercas vivas, combustible, obtención de principios con interés medicinal, insecticidas y extracción de aceites y grasas, además de que las leguminosas presentan un beneficio adicional como mejoradores del suelo en cuanto a su fertilidad, ya que sus nódulos radiculares tienen la propiedad de fijar el nitrógeno atmosférico. Por otra parte no dudamos que el futuro se sumen nuevas especies de leguminosas a este selecto grupo de plantas, y se pueda contar con información apropiada sobre los insectos que regulan las poblaciones de las plantas será de gran importancia.

Agradecimientos. Al Doctor Óscar Dorado Ramírez del Centro de Educación Ambiental e Investigación de la Sierra de Huautla (CEAMISH-UAEM), por su colaboración en la revisión de las plantas hospederas de brúquidos con nuevo registro para el estado de Morelos.

\section{LITERATURA CITADA}

Burgos Solorio, A. \& Trejo Loyo, A. G. 2001. Lista Preliminar de los Coleópteros Registrados para el estado de Morelos, pp. 67-95. In: J.L. Navarrete Heredia, H.E. Fierro López \& A. Burgos Solorio (Eds.). Tópicos sobre Coleoptera de México. Universidad de Guadalajara y Universidad Autónoma del Estado de Morelos.

De la Cruz P., A., Romero N., J., Carrillo S., J. L., García L., E., Grether G., R., Sánchez S., S. \& Pérez de la C., M. 2013. Brúquidos (Coleoptera: Bruchidae) del estado de Tabasco, México. Acta Zoológica Mexicana (n.s.), 29: 1-95.

De Loera B., J. C., Romero N., J., Valdez C., J. \& Carrillo S., J. L. 2006. Especies y hospederas de los Bruchidae (Insecta: Coleoptera) del estado de Jalisco, México. Agrociencia, 40: 511-520.

García, E. 1973. Modificaciones al Sistema de Clasificación Climática de Köppen. Instituto de geografía, UNAM, México $2^{\text {a }}$ Edición.

Johnson, C. D. 1990. Six new species of Acanthoscelides from North and Central America (Coleoptera: Bruchidae). The Coleopterist Bulletin, 44: 3-18.

Johnson, C. D. \& Kingsolver, J. M. 1973. A revision of the genus Sennius of North and Central America (Coleoptera: Bruchidae). United States Department of Agriculture, Technical Bulletin no. 1462: $135 \mathrm{pp}$.

Johnson, C. D. \& Kingsolver, J. M. 1976. Systematics of Stator of North and Central America (Coleoptera: Bruchidae). United States Department of Agriculture, Technical Bulletin no. 1537, 101 pp.

Johnson, C. D., Southgate, J. B. \& Delobel, A. 2004. A revision of the Caryedontini (Coleoptera: Bruchidae: Pachymerinae) of Africa and the Middle East. Memoirs of the American Entomological Society, 44: 1-126.

Kingsolver, J. M. 1970. A study of male genitalia in Bruchidae (Coleoptera). Proceedings of the Entomological Society of Washington, 72: 370-386.

Kingsolver, J. M. 1988. Biosystematics of the genus Merobruchus of continental North America and the West Indies (Coleoptera: Bruchidae). U.S. Department of Agriculture, Technical Bulletin no. 1744: 1-63.

Kingsolver, J. M. 2004. Handbook of the Bruchidae of the United States and Canada (Insecta: Coleoptera). U. S. Department of Agriculture, Technical Bulletin no. 1912, Vol. 1 \& 2: 324 pp. 
Kingsolver, J. M. \& Johnson, C. D. 1978. Systematics of the genus Mimosestes (Coleoptera: Bruchidae). United States Department of Agriculture, Technical Bulletin no. 1590: 1-106.

Kingsolver, J. M. \& Whitehead, D. R. 1974a. Biosystematics of Central American species of Ctenocolum, a new genus of seed beetles (Coleoptera: Bruchidae). Proceedings of the Biological Society of Washington, 87: 283-312.

Kingsolver, J. M. \& Whitehead, D. R. 1974b. Classification and comparative biology of the seed beetle genus Caryedes Hummel (Coleoptera: Bruchidae). Transactions of the American Entomological Society, 100: 341-430.

Kingsolver, J. M. \& Whitehead, D. R. 1976. The North and Central American species of Meibomeus (Coleoptera: Bruchidae: Bruchinae). United States Department of Agriculture, Technical Bulletin no. 1523: 1-54.

Luna, C. J., Romero N., J. \& Jones, R. W. 2002. Listado de Bruchidae del Estado de Querétaro, México (Insecta: Coleoptera). Acta Zoológica Mexicana (n.s.), 87: 17-28.

Luna L., C., Morales, N. O. \& Romero N, J. 2006. Brúquidos (Coleoptera: Bruchidae) de Platanillo, Gro, pp.1054-1057. In: G. Estrada V., J. Romero Nápoles E., A. Equihua M., C. Luna L. y J. L. Rosas A. (Eds.). Entomología Mexicana. Vol. 5, Tomo 2. Colegio de Postgraduados. Montecillo, Edo de México.

Nadal, M. S., Moreno Y., M. T. \& Cubero S., J. I. 2004. Las leguminosas grano en la agricultura moderna. Junta de Andalucía, Consejería de Agricultura y pesca. Madrid.

Romero G., G. 2013. Estudio Faunístico de la Familia Bruchidae (Insecta: Coleóptera) del Estado de Morelos, México. Colegio de postgraduados. Tesis de Doctorado. Programa de Fitosanidad, Entomología y Acarologia. 192 pp.

Romero N., J. 2002a. Bruchidae, pp. 513-534. In: J. Llorente Bousquets \& J. J. Morrone (Eds.). Biodiversidad, taxonomía y biogeografía de artrópodos de México: Hacia una síntesis de su conocimiento. UNAM, Vol. III.

Romero N., J. 2002b. Five new species of Meibomeus Bridwell from the New World with host records for them and six named species (Coeloptera: Bruchidae). The Coleopterist Bulletin, 56: 182-202.

Romero N., J. 2011. Gorgojos de las semilla (Insecta: Coleoptera: Bruchidae), pp. 361-365. In: Comisión Nacional para el Conocimiento y Uso de la Biodiversidad (CONABIO). La biodiversidad en Veracruz: Estudio de Estado. Comisión Nacional para el Conocimiento y Uso de la Biodiversidad. Gobierno del Estado de Veracruz, Universidad Veracruzana, Instituto de Ecología, A.C. México. Apéndice VIII.27, 1-4.

Romero N., J. \& Johnson, C. D. 1999. Zabrotes sylvestris, a new species from the United States and Mexico related to Z. subfasciatus (Boheman) (Coleoptera: Bruchidae: Amblycerinae). The Coleopterist Bulletin, 53: 87-98.

Romero N., J. \& Johnson, C. D. 2000. Revision of the genus Zabrotes Horn of Mexico (Coleoptera: Bruchidae: Amblycerinae). Transactions of the American Entomological Society, 126: 221-274.

Romero N., J. \& Johnson, C. D. 2002a. BRUCOL, una base de datos para Bruchidae (Insecta: Coleoptera), pp. 520-524. In: J. Romero Nápoles, E. G. Estrada V. \& A. Equihua M (Eds.). Entomología Mexicana, Vol. 1. Colegio de Postgraduados-SME. Montecillo, Edo de México.

Romero N., J. \& Johnson, C. D. 2002b. Cassia moschata H.B.K., New Host for Caryedon serratus (Olivier) in the New World (Coleoptera: Bruchidae: Pachymerinae). The Coleopterists Bulletin, 56: 95-96.

Romero N., J. \& Jonhson, C. D. 2003. Meganeltumius juani, New Genus, New Species (Coleoptera: Bruchidae). The Coleopterists Bulletin, 57: 237-242.

Romero N., J. \& Johnson, C. D. 2004. Sinopsis de los brúquidos de México (Insecta: Coleoptera), pp. 758-763. In: Morales M., A. M. Ibarra G., A. del P. Rivera G. \& S. Stanford C. (Eds.). Entomología 
Mexicana, Vol. 3. Colegio de Postgraduados-SME. Montecillo, Edo de México.

Romero N., J., Johnson, C. D. \& Kingsolver, J. M. 1996. Revision of the genus Amblycerus of the United States and México (Coleoptera: Bruchidae: Amblycerinae). United States Department of Agriculture, Technical Bulletin no. 1845. 166 pp.

Romero N., J. \& Westcott, R. L. 2011. The Bruchidae (Insecta: Coleoptera) of La Reserva de la Biósfera Sierra de Huautla, Morelos, Mexico, with descriptions of two new species and an annotated checklist. Insecta Mundi, 0166: 1-15.

Rzedowski, J. 1978. Vegetación de México. Editorial Limusa, México.

Terán, A. L. \& Kingsolver, J. M. 1977. Revisión del género Megacerus (Coleoptera: Bruchidae). Opera Lilloana, 25: 1-287.

Vidal, Z. R. 1980. Algunas relaciones Clima-Cultivos en el Estado de Morelos. Instituto de geografía. Universidad Nacional Autónoma de México. México, Distrito Federal.

Whitehead, D. R. \& Kingsolver, J. M. 1975. Biosystematics of the North and Central American Species of Gibbobruchus (Coleoptera: Bruchidae: Bruchinae). Transactions of the American Entomological Society, 101: 166-225. 